\title{
Correlation between a Change of Drug Resistance of Klebsiella pneumoniae and Defined Daily Doses of Antimicrobial Agents from 2014 to 2018
}

\author{
Jiuli Hu, Xu Xiao, Chanchan Hu, Rui Wang, Yanwu Zhao, Xiaoqin Zhu* \\ Affiliated Hospital of ChengdeMedical University, Chegnde, Hebei, China \\ Email: *cdzhuxiaoqin@aliyun.com
}

How to cite this paper: Hu, J.L., Xiao, X., Hu, C.C., Wang, R., Zhao, Y.W. and Zhu, X.Q. (2020) Correlation between a Change of Drug Resistance of Klebsiella pneumoniae and Defined Daily Doses of Antimicrobial Agents from 2014 to 2018. Pharmacology \& Pharmacy, 11, 299-305. https://doi.org/10.4236/pp.2020.1111024

Received: September 21, 2020

Accepted: November 9, 2020

Published: November 12, 2020

Copyright $\odot 2020$ by author(s) and Scientific Research Publishing Inc. This work is licensed under the Creative Commons Attribution International License (CC BY 4.0).

http://creativecommons.org/licenses/by/4.0/

(c) (i) Open Access

\begin{abstract}
Introduction: The prevalence of Klebsiella pneumoniae has rapidly increased in recent years and the distribution differed greatly by region, We aimed to study the relationship between antibiotic resistance and $K$. pneumoniae, especially carbapenem-resistant Klebsiella pneumoniae (CRKP) in our tertiary hospitals from 2014 to 2018. Methodology: The antibiotic consumption data of $K$. pneumoniae were expressed as the defined daily dose (DDD) per 100 inpatient days (DDDs). K. pneumoniae which isolated from clinical samples in hospital between January 2014 and December 2018 were retrospectively analyzed, and the correlation between antibiotic resistance rate and antibiotic frequency was analyzed. Results: From 2014 to 2018, a total of 2295 strains of $K$. pneumoniae were isolated, with the detection rates of $8.2 \%$, $9.2 \%, 11.9 \%, 13.4 \%$ and $14.0 \%$. There were 423 strains of CRKP, with the detection rates of $7.5 \%, 5.8 \%, 17.8 \% 24.2 \%$ and $25.2 \%$ respectively. $K$. pneumoniae showed different degrees of resistance to antibiotics and showed an increasing trend year by year to carbapenems. The resistance rate of imipenem was $2.5 \%, 2.8 \%, 9.9 \%, 12.3 \%, 13.4 \%$, and the resistance rate of meropenem was $2.0 \%, 3.0 \%, 8.8 \%, 12.6 \%, 12.7 \%$, respectively. The resistance rate of most other drugs decreased. The DDDs values of cefoperazone/sulbactam, piperacillin-tazobactam and gentamicin showed a strong positive correlation with $K$. pneumoniae drug resistance rate $(\mathrm{r}>0.8, \mathrm{P}<0.05)$. Conclusions: The detection rate of $K$. pneumoniae and CRKP increased year by year, which was closely related to the dose of antibiotics. Strengthening the management of antimicrobial drugs and standardising the use of antimicrobial prescriptions were of great significance for delaying the emergence of drug-resistant bacteria.
\end{abstract}


Keywords

Klebsiella pneumonia, Antibiotic Consumption, Resistance, Correlation Analysis, Defined Daily Doses

\section{Introduction}

Klebsiella pneumoniae is a gram-negative bacillus, which is an essential condition pathogenic bacterium and iatrogenic infectious bacterium in Enterobacteriaceae Klebsiella. In recent years, the bacterial resistance rate has been increasing with the widespread use of antibacterial drugs and the rapid change of bacteria, especially carbapenem-resistant $K$. pneumoniae (CRKP), CHINET data showed that the detection rate of CRKP was $13.8 \%$ in 2007 [1], increasing to $25.0 \%$ and $26.3 \%$ in 2018 [2]. Therefore, the rational use of antibacterial drugs has become an essential means of curbing bacterial resistance. This study monitored and analysed the drug resistance of $K$. pneumoniae in the hospital from January 2014 to December 2018, and at the same time analysed the frequency of use of antimicrobial drugs among inpatients during this period to provide a basis for the rational use of antimicrobial drugs.

\section{Methodology}

\subsection{Sample Source and Antimicrobial Testing}

Klebsiella pneumoniae were isolated from various patient bodily fluids/sites including sputum, urine, pus, blood, sterile body fluid, surgical wound, submitted by the clinical department of the hospital from 2014 to 2018. The samples to exclude the same patient during a hospital and the same strain of the same part were separated and identified by Vitek-2 system (French bio-Merieux company) and the K-B method following guidelines recommended by the Clinical and Laboratory Standards Institute (CLSI), 2011 (M100-S21) [3]. The quality control strain was Klebsiella pneumoniae ATCC 700603, which was purchased from the Clinical Laboratory of the Ministry of Health.

\subsection{Detecting of the Carbapenem-Resistant $K$. pneumoniae (CRKP)}

The modified Hodge test (MHT) was used to dilute the suspension of $K$. pneumoniae ATCC $7006030.5 \mathrm{M}$. turbidity bacteria with sterile normal saline at 1:10 and evenly spread on the $\mathrm{MH}$ plate. After drying at room temperature for 3 - 10 min, meropenem was pasted in the middle, and the bacteria to be tested were streaked from the edge of the paper sheet to the edge of the plate. The results were observed after $18-24 \mathrm{~h}$ incubation at $35 \mathrm{C}$. Result: when the MIC of meropenem $\geq 2 \mathrm{ug} / \mathrm{ml}$, it was a carbapenem-resistant strain.

\subsection{Data Collection and Antimicrobial Consumption}

The antibacterial drug data was derived from the hospital HIS systems from 
January 2014 to December 2018. The antibacterial drug distribution information of the inpatient pharmacy and the relevant information in Excel. The total consumption is calculated and using the defined daily dose (DDD) classification recommended by the World Health Organization (WHO)

(http://www.whocc.no/atc ddd index/). Inpatient days were calculated by multiplying the quarterly total number of hospital discharges with the mean number of days of hospitalization [4]. Antimicrobial agents tracked include aminoglycosides, $\beta$-lactam antibiotics (penicillins, cephalosporins, carbapenems), quinolones, sulfa antibacterial drugs.

\subsection{Statistical Analysis}

The trend over time for antimicrobial consumption and rates of KP was calculated independently by Pearson's correlation analysis. The Pearson's correlation coefficient ( $r$ ) between the bacterial resistance rate and the DDDs value of antibacterial drugs was calculated: $r>0.8$, which was considered to have a strong correlation between them; $r<0.3$, the relationship between them is considered to be weak; $\mathrm{P}<0.05$ is considered statistically significant. All statistical analyses were performed using IBM SPSS Statistics 24.0.

\section{Results}

\subsection{Detection Rate of Klebsiella pneumoniae}

From 2014 to 2018, a total of 2295 strains of $K$. pneumoniae were detected, and the detection rate increased from $8.3 \%$ in 2014 to $14.1 \%$ in 2018 , showing an increasing trend year by year. Among them, 423 CRKP strains were detected, and the isolation rate was from $7.3 \%$ in $2014,5.8 \%$ in 2015 to $25.2 \%$ in 2018 . The specific data are shown in Table 1.

\subsection{The Distribution of Species}

A total of $2295 \mathrm{~K}$. pneumoniae were isolated from sputum, urine and pus, and their composition ratios were $67.2 \%, 12.3 \%$ and $7.8 \%$, respectively. But CRKP was mainly derived from sputum, blood and urine, with a composition ratio of $50.4 \%, 13.9 \%$ and $12.8 \%$, respectively. The specific data are shown in Table 2.

\subsection{Antibacterial Drugs Resistance Rate of $K$. pneumoniae and Its Correlation with DDDs}

$K$. pneumoniae have different degrees of resistance to antibacterial drugs, and the resistance to carbapenems has been a significant increasing trend. From 2014 to 2018 , The resistance rates of imipenem are $2.53 \%, 2.80 \%, 9.90 \%, 12.30 \%$ and $13.41 \%$, the drug resistance rates of meropenem were $2.04 \%, 3.00 \%, 8.80 \%$, $12.60 \%$, and $12.72 \%$, respectively. The drugs with the highest DDDs value were cefoperazone/sulbactam and showed an upward trend each year. The smaller values were ciprofloxacin and imipenem. The correlation between the resistance rate of KP to common antibacterial drugs and the changes in the corresponding antibacterial drugs DDDs value from 2014 to 2018 is shown in Table 3. 
Table 1. The isolation rates of Klebsiella pneumoniae (KP) and CRKP strains from 2014 to 2018 (\%).

\begin{tabular}{|c|c|c|c|c|c|}
\hline \multirow{2}{*}{ Year } & \multirow{2}{*}{$\mathrm{n} /$ Strain } & \multicolumn{2}{|c|}{ KP } & \multicolumn{2}{|c|}{ CRKP } \\
\hline & & Strain & Rate (\%) & Strain & Rate (\%) \\
\hline 2014 & 3572 & 292 & 8.2 & 22 & 7.4 \\
\hline 2015 & 3561 & 329 & 9.2 & 19 & 5.8 \\
\hline 2016 & 3866 & 461 & 11.9 & 82 & 17.8 \\
\hline 2017 & 4156 & 556 & 13.4 & 135 & 24.3 \\
\hline 2018 & 4662 & 650 & 14 & 165 & 25.2 \\
\hline Total & 19,817 & 2288 & 11.5 & 423 & 18.5 \\
\hline
\end{tabular}

Table 2. Distribution and percentage of KP and CRKP by clinical specimens (\%) (2014-2018).

\begin{tabular}{ccccc}
\hline \multirow{2}{*}{ Strain source } & \multicolumn{2}{c}{ KP } & \multicolumn{2}{c}{ CRKP } \\
\cline { 2 - 5 } & Strain $(\mathrm{n}=2295)$ & Rate (\%) & Strain (n=423) & Rate (\%) \\
\hline sputum & 1542 & 67.2 & 213 & 50.4 \\
urine & 283 & 12.3 & 54 & 12.8 \\
pus & 179 & 7.8 & 40 & 9.5 \\
sterile body fluid & 125 & 5.4 & 29 & 6.9 \\
blood & 103 & 4.5 & 59 & 13.9 \\
surgical wound & 63 & 2.7 & 28 & 6.4 \\
\hline
\end{tabular}

Table 3. Drug resistance rate (\%) of the KP strains to the commonly used antbiotics and relationship with the changes of DDDs of antibiotics.

\begin{tabular}{|c|c|c|c|c|c|c|c|c|c|c|c|c|}
\hline \multirow{2}{*}{ Antibacterial drugs } & \multicolumn{5}{|c|}{ Drug resistance rate of KP (\%) } & \multicolumn{5}{|c|}{ DDDs } & \multirow{2}{*}{$\mathrm{r}$} & \multirow{2}{*}{$\mathrm{P}$} \\
\hline & 2014 & 2015 & 2016 & 2017 & 2018 & 2014 & 2015 & 2016 & 2017 & 2018 & & \\
\hline Ampicillin & 100 & 99.6 & 100 & 99.8 & 99.7 & - & - & - & - & - & - & - \\
\hline Ciprofloxacin & 23.8 & 23.5 & 26.5 & 30.5 & 30.4 & 185.5 & 172.4 & 147.2 & 132.8 & 165.8 & -0.682 & 0.205 \\
\hline Ceftriaxone & 56.6 & 54.6 & 62.9 & 47.2 & 46.3 & 6831 & 7184 & 9374 & 14634 & 15494 & -0.772 & 0.126 \\
\hline Cefoperazone/sulbactam & 10.8 & 9.8 & 13.9 & 20.5 & 22.3 & 25,642 & 27,283 & $36,402.7$ & $41,241.4$ & $46,848.6$ & 0.964 & 0.008 \\
\hline Cefuroxime & 62.5 & 59.5 & 73.4 & 49.2 & 48 & 465.3 & 495.7 & 2221 & 5105.7 & 5865.5 & -0.697 & 0.191 \\
\hline Cefazolin & 65.6 & 62.6 & 54.4 & 54.3 & 58.5 & 5869.2 & 5511.7 & 4627.3 & 9294 & 10,684 & -0.277 & 0.651 \\
\hline Cefoxitin & 31.6 & 29.6 & 35.9 & 22.1 & 23.1 & - & - & - & - & - & - & - \\
\hline Gentamicin & 43.4 & 42.4 & 30.4 & 22.6 & 21.9 & 4754.8 & 4563.3 & 3024.3 & 2989.7 & 2579.6 & 0.967 & 0.007 \\
\hline Imipenem & 2.5 & 2.8 & 9.9 & 12.3 & 13.4 & 123.8 & 132.5 & 389.3 & 306.8 & 314.4 & 0.873 & 0.053 \\
\hline Levofloxacin & 25.2 & 22.2 & 23.9 & 26 & 25.9 & $15,483.7$ & $16,539.8$ & $21,092.4$ & $20,170.4$ & $25,367.5$ & 0.48 & 0.413 \\
\hline Meropenem & 2 & 3 & 8.8 & 12.6 & 12.7 & 6537.9 & 4970.8 & 6750.7 & $10,914.4$ & $12,835.8$ & 0.876 & 0.052 \\
\hline Piperacillin/tazobactam & 9.4 & 10.4 & 13.2 & 17.1 & 17.2 & 2146.6 & 2395.6 & 5356.2 & 6733.5 & 7483.4 & 0.98 & 0.003 \\
\hline Fuxin Xinming & 46.5 & 48.5 & 42.7 & 28.1 & 27.6 & - & - & - & - & - & - & - \\
\hline
\end{tabular}

Note: - indicates that this item has no data. 


\subsection{Correlation Analysis of the Changing Trend of Antibacterial Drugs DDDs Value and Resistance Rate of $K$. pneumoniae}

The strength of association between DDDs of cefoperazone/sulbactam, piperacillin/tazobactam, gentamicin and resistance rate of $K$. pneumoniae were very high $(r=0.964,0.980,0.967)$, and that the correlation coefficient is very highly significantly different from zero $(\mathrm{P}<0.01)$. The values of DDDs of amikacin and ciprofloxacin were negatively correlated with the resistance rate of $K$. pneumoniae $(\mathrm{r}=-0.965,-0.972, \mathrm{P}<0.05)$. The DDDs of levofloxacin had a correlation with the $K$. pneumoniae resistance rate of $0.8>\mathrm{r}>0.6, \mathrm{P}<0.01$. The DDDs of meropenem had a strong positive correlation with $K$. pneumoniae resistance rate $\mathrm{r}>0.8$, but $\mathrm{P}>0.05$ showed no difference. Imipenem $0.5<\mathrm{r}<0.8, \mathrm{P}>0.05$, its correlation is not large. For other antibacterial drugs, $\mathrm{r}<0.8, \mathrm{P}>0.05$, the correlation is not great.

\section{Discussion}

Our study highlights the rising resistance rates of $K$. pneumoniae which isolated our hospital from 2014 to 2018, especially the resistance rates of CRKP has rapidly increased from $7.5 \%$ in 2014, 5.8\% in 2015 and $17.8 \%$ in 2016, 24.2\% in 2017 to $25.2 \%$ in 2018 . In our study our hospital should pay attention to the resistance of CRKP because carbapenems are the last line in defence against the pathogen, clinical drug treatment is facing severe challenges and changes, and it is a wake-up call for clinicians and pharmacists. This article wants to provide valid data for the clinic by counting the correlation between the drug resistance rate of bacteria and the frequency of use of antibacterial drugs DDDs. Reasonable norms, to eliminate the phenomenon of unindicated medication and medication for too long [5].

Antimicrobial resistance (AMR) is currently one of the most urgent public health issues in the world [6]. The resistance of $K$. pneumoniae is mediated by a series of mechanisms, which are mainly related to the widespread use of broad-spectrum antibacterial drugs. Catalytic hydrolysis to produce KPC carbapenemase or NDM-1 metalloenzyme can hydrolyze carbapenems to produce CRKP. Resistance to other antibacterial drugs is related to the combined action of biofilm formation, loss or alteration of membrane pore proteins, and efflux pumps [7] [8] [9].

From the data, it was found that 15 antibacterial drugs of $K$. pneumoniae were tested for in vitro susceptibility. The increase in drug resistance rate was directly related to the amount of clinical use. The DDDs of cefoperazone/sulbactam rose from $27,283.0$ to $41,241.4$. The resistance rate of cefoperazone/sulbactam to $K$. pneumoniae increased from $9.8 \%$ to $20.5 \%$, and the DDDs of piperacillin sodium/tazobactam sodium increased from 2395.6 to 6733.5 and $K$. pneumoniae to cefoperazone/sulbactam The rate increased from $10.43 \%$ to $17.09 \%$, and the DDDs of gentamicin decreased from 4563.3 to 2989.7 , which was highly corre- 
lated with the resistance rate of $K$. pneumoniae to gentamicin from $42.4 \%$ to $22.6 \%(\mathrm{r}=0.953,0.938,0.928, \mathrm{P}<0.05)$; the values of DDDs of amikacin and ciprofloxacin were strongly negatively correlated with the resistance rate of $K$. pneumoniae $(\mathrm{r}=-0.965,-0.972, \mathrm{P}<0.05)$. The meropenem DDDs values were not significantly correlated with $K$. pneumoniae drug resistance rate $(\mathrm{r}>0.8, \mathrm{P}>$ $0.05)$. Imipenem $0.5<\mathrm{r}<0.8, \mathrm{P}>0.05$, its correlation is not large. It is suggested that the emergence of bacterial resistance is not only related to antibacterial drugs DDDs but may also be related to factors such as cross-resistance of bacteria. Among them, the widespread use of broad-spectrum $\beta$-lactamase inhibitors affects the effect of carbapenem drugs on the Antibacterial activity of $K \cdot$ pneumoniae. Zhan [10] studies have consistent with the study of the exhibition champion. Piperacillin/tazobactam DDDs are highly correlated with imipenem resistance. Meng [11] studies considered that Piperacillin tazobactam, cefoxitin, imipenem, Meropenem and amikacin are still the first choice for the treatment of $K$. pneumoniae infection. because the drug resistance rate of $K$. pneumoniae to piperacillin was significantly positively correlated with AUD of ceftazidime. The drug resistance rate of imipenem was positively correlated with AUD of amoxicillin clavulanate.

In the study, the monitoring data shows that the resistance of $K$. pneumoniae to carbapenem antibacterial drugs is on the rise because carbapenem antibacterial drugs are the most effective antibacterial drugs for the treatment of $K$. pneumoniae infections [8]. Therefore, Our hospital establishes an fore-warning mechanism for different bacteria every quarter to cope with the difficult drug resistance situation and improving the level of anti-infective treatment and controlling the clinical application of antibacterial drugs refers to the Guidelines for the Clinical Application of Antibacterial Drugs to increase the rate of clinical specimen submission and detection, Choose appropriate antibacterial drugs according to the susceptibility of bacteria.

After 5-year period analysis of drug resistance rates, although $K$. pneumoniae has aminoglycosides (amikacin), enzyme-inhibitors (piperacillin/tazobactam, cefoperazone/sulbactam), The sensitivity of cephalosporins (cefoxitin) and carbapenems (imipenem, meropenem) has remained at a high level, and the resistance rate of the third and fourth generation cephalosporins has increased, especially CRKP is gradually increasing. Therefore, the drug resistance rate of $K$. pneumoniae is highly correlated with the use of various antibacterial drugs. Rotation of antibacterial drugs can reduce the drug resistance rate of bacteria.

\section{Conclusion}

Our data demonstrate a significant increase in the prevalence of $K$. pneumoniae and the consumption of enzyme-inhibitors in our hospital. We found that the high incidence of CRKP is associated with increased enzyme-inhibitors usage in our hospital. Future work is needed to elucidate the factors which influence the increased consumption of enzyme-inhibitors usage in our hospital. 


\section{Funding}

This work was funded by the Chengde Science and Technology Planning Project (No. 201701A094).

\section{Conflicts of Interest}

The authors declare no conflicts of interest regarding the publication of this paper.

\section{References}

[1] Wang, F., Zhu, D.M., Hu, F.P., et al. (2008) CHINET Surveillance of Bacterial Resistance in China: 2007 Report. Chinese Journal of Infection and Chemotherapy, 8 , 325-333.

[2] Hu, F.P., Guo, Y., Zhu, D.M., et al. (2020) CHINET Surveillance of Bacterial Resistance in China: 2018 Report. Chinese Journal of Infection and Chemotherapy, 20, $1-10$.

[3] Clinical and Laboratory Standards Institute (CLSI) (2011) Performance Standards for Antimicrobial Susceptibility Testing; Twenty-Third Informational Supplement (M100-S21).

[4] Sun, J., Shen, X., Li, M., et al. (2015) Changes in Patterns of Antibiotic Use in Chinese Public Hospitals (2005-2012) and a Benchmark Comparison with Sweden in 2012. Journal of Global Antimicrobial Resistance, 3, 95-102. https://doi.org/10.1016/j.jgar.2015.03.001

[5] Xia, M.Y., Gao, Q.C. and Yuan, C.G. (2014) Change of Drug Resistance of Escherichia Coli and Klebsiella pneumoniae, Chinese Journal of Nosocomiology, 24, 287-289.

[6] Brady, M., Cunney, R., Murchan, S., Oza, A. and Burns, K. (2016) Klebsiella pneumoniae Bloodstream Infection, Antimicrobial Resistance and Consumption Trends in Ireland: 2008 to 2013. European Journal of Clinical Microbiology \& Infectious Diseases, 35, 1777-1785. https://doi.org/10.1007/s10096-016-2727-4

[7] Yu, W.L., Guo, Y.X., Li, X., et al. (2017) Current Status and Mechanisms of Imipenem Resistant Klebsislla pneumoniae. Chinese Journal of Hospital Pharmacy, 37, 2210-2215.

[8] Gong, X., Wang, Y., Zhang, J.S., et al. (2018) Resistance Mechanism and Homology Analysis of Klebsiella pneumoniae Resistance. Chinese Journal of Clinical Laboratory Science, 36, 270-273.

[9] Piperaki, E.T., George, A., Leonidas, S., et al. (2017) Klebsiella pneumoniae: Virulence, Biofilm and Antimicrobial Resistance. The Pediatric Infectious Disease Journal, 36, 1002-1005. https://doi.org/10.1097/INF.0000000000001675

[10] Zhan, G.J., Shao, H. and Zhang, L. (2014) Analysis of Drug Resistance and the Correlation of Drug Resistance to Klebsiella pneumoniae in Our Hospital from 2009-2013. China Pharmacy, 25, 4349-4355.

[11] Meng, G.Y., Wangl, D.X., Zhong, L.Q., et al. (2019) Correlation between Antimicrobial Resistant Rate of Klebsiella pneumoniae and Antimicrobial Use Density. Chinese Journal of Hospital Pharmacy, 39, 1639-1644. 